\title{
TITLE:
}

\section{Polymerization reactions in porous coordination polymers}

$\operatorname{AUTHOR}(\mathrm{S}):$

Uemura, Takashi; Yanai, Nobuhiro; Kitagawa, Susumu

CITATION:

Uemura, Takashi ...[et al]. Polymerization reactions in porous

coordination polymers. Chemical Society reviews 2009, 38(5): 1228 -

1236

ISSUE DATE:

2009-05

URL:

http://hdl.handle.net/2433/123401

\section{RIGHT:}

Chem. Soc. Rev., 2009, pp.1228-1236 - Reproduced by permission of The Royal Society of Chemistry (RSC); この論文は出版社版でありません。引用の際には出版社版をご確認ご 利用ください。; This is not the published version. Please cite only the published version. 


\title{
Polymerization Reactions in Porous Coordination Polymers
}

\author{
Takashi Uemura, ${ }^{a, b}$ Nobuhiro Yanai ${ }^{a}$ and Susumu Kitagawa* ${ }^{a, c}$ \\ Received (in XXX, XXX) 1st January 2007, Accepted 1st January 2007 \\ First published on the web 1st January 2007 \\ ${ }_{5}$ DOI: 10.1039/b000000x
}

Recent developments of polymerizations within the nanochannels of porous coordination polymers (PCPs) are reviewed. The characteristic features of PCPs are regular structures, controllable channel sizes and shapes, designable surface functionality, and flexible frameworks, which can be utilized for precision polymer synthesis and specific polymer confinement. This tutorial review 10 discusses promising approaches to multiple controls of polymer structures, analysis systems for low-dimensional polymer assemblies, and the construction of PCP-polymer nanohybrid, which is a strong enticement to researchers in the areas of inorganic, materials, and polymer chemistry.

\section{Introduction}

One of the outstanding challenges in polymer materials 15 science is the fabrication of systems that allow the controlled arrangement of monomers to be polymerized to materials useful for a desired purpose. ${ }^{1,2}$ All naturally occurring polymers in the biological events are produced by enzymatic catalysis, where stereoselective, regioselective, and 20 chemoselective polymerizations proceed within regulated and well-organized molecular-scale spaces. In the biomineralization process, biominerals (inorganic polymers), generally consisting of either calcium, iron, or silicon, are produced in living organisms. In attempts to mimic these ${ }_{25}$ elegant systems, scientists have utilized channel structures, layered materials, or nanolevel templates, such as zeolites, clays, and organic hosts and polymers, for the preparation of functional polymeric materials with controlled structures, which have shown specific nanospace effects on the reaction 30 kinetics and selectivity in the inclusion polymerization processes. ${ }^{3}$ Preparation of host-guest nanocomposites is also of interest for nanomaterial synthesis, host-guest synergistic properties, and nanosize-dependent properties. ${ }^{4}$

Polymeric coordination frameworks with microporous 35 regular channel structures, which are regarded as metalorganic frameworks (MOFs) or porous coordination polymers (PCPs), are promising materials for applications in catalysis, separation, gas storage, and molecular recognition. ${ }^{5}$ In this area, the structural versatility of molecular chemistry has 40 allowed the rational design and assembly of materials having novel topologies and exceptional host-guest properties. Compared with conventional porous matrices, PCPs are of particular interest because of their designable framework structures; size, shape, dimension, and surface functionality of 45 the nanochannels can be systematically tuned by changing the combination between organic ligands and metal ions (Fig. 1). ${ }^{5}$ These features are attractive enough to attract our efforts toward the development of PCPs for applications in polymer materials science. ${ }^{2}$ Polymerization of monomers encapsulated 50 within confined and designed nanospaces based on PCPs can lead to polymeric materials with our desired structures, which can be regarded as tailor-made polymerization systems. ${ }^{2}$ In this review, we will discuss the recent progress and future perspectives of various polymerization systems utilizing the ${ }_{55}$ PCP nanochannels for controlling primary and secondary polymer structures as well as for obtaining specific properties based on low-dimensional assemblies of polymers and functional polymer-based nanohybrids.

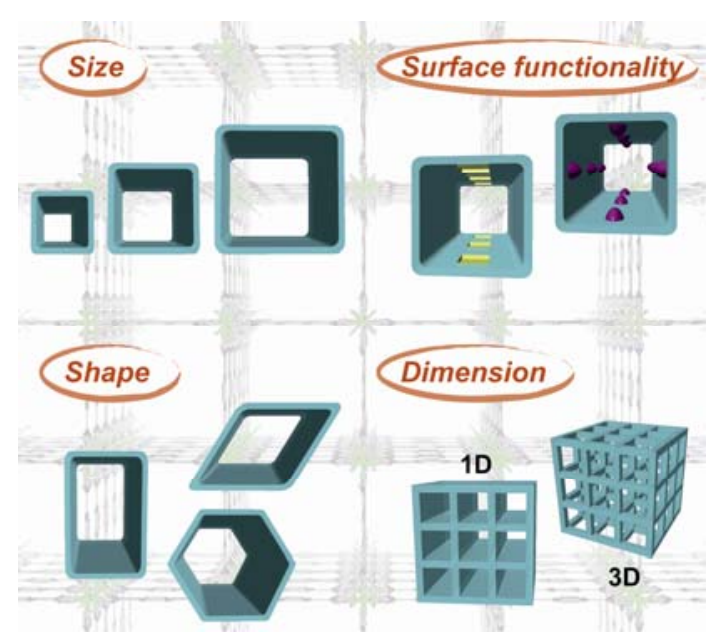

Fig. 1 Designable channel features of PCPs from the viewpoints of pore size, shape, dimension and surface functionality.

\section{Radical polymerization in PCPs}

60 Radical polymerization has been one of the most efficient methods for preparation of a massive amount of commercially available polymer materials because of the versatility of the polymerizable monomers and the stable nature of the polymerizations to water or moisture and polar and ionic ${ }_{65}$ compounds. ${ }^{6}$ However, precision polymer synthesis in radical polymerization is very difficult because of the highly reactive free radical species, which lead to rapid chain propagation without control of stereoselectivity and undergoes inevitable termination via radical-radical coupling and 70 disproportionation. Therefore, the advent of effective methods for taming radical polymerizations has long been sought for controlling molecular weight, stereoregularity (tacticity), reaction sites, copolymer sequence, and so on. ${ }^{7-11}$ 
To attain precise control of those polymer primary structures, radical polymerization of vinyl monomers was performed in various nanochannels of $\left[\mathrm{M}_{2}(\mathrm{~L})_{2} \text { ted }\right]_{n}$ (ted = triethylenediamine, 1a, $\mathrm{M}=\mathrm{Cu}^{2+}, \mathrm{L}=$ biphenyl-4,4'5 dicarboxylate; $\mathbf{1 b}, \mathrm{M}=\mathrm{Cu}^{2+}, \mathrm{L}=1$,4-benzenedicarboxylate; 1c, $\mathrm{M}=\mathrm{Cu}^{2+}, \mathrm{L}=1$,4-naphthalenedicarboxylate; $\mathbf{2 b}, \mathbf{M}=$ $\mathrm{Zn}^{2+}, \mathrm{L}=1$,4-benzenedicarboxylate; $2 \mathrm{c}, \mathrm{M}=\mathrm{Zn}^{2+}, \mathrm{L}=1,4-$ naphthalenedicarboxylate) (Fig. 2). ${ }^{12-15}$
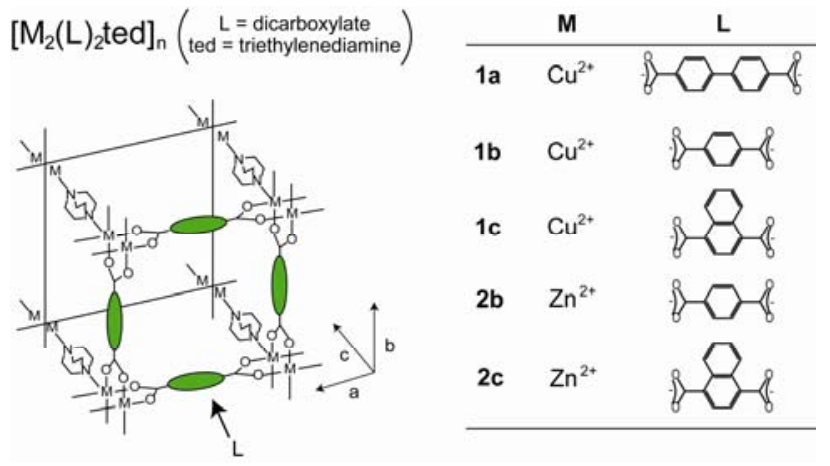

Fig. 2 PCP structures of 1 and 2.

\subsection{Control of molecular weight}

10 Recently, living radical polymerization of vinyl monomers in solution systems has emerged as an effective method for precision vinyl polymer synthesis, because this polymerization is free from side reactions, such as termination and chain transfer, resulting from the introduction of dormant

15 species in the growing chain terminal, and can thus control the molecular weights. ${ }^{7-10}$

Radical polymerization of vinyl monomers, such as styrene (St) and methyl methacrylate (MMA) in the nanochannels of $\mathbf{1}$ and $\mathbf{2}$ showed a 'living radical' nature, where the propagating 20 radicals in this system were remarkably stabilized because of effective entrapment in the nanochannels. ${ }^{12,14}$ This was shown by the electron spin resonance (ESR) measurements for propagating radicals of polystyrene (PSt) and poly(methyl methacrylate) (PMMA) in the nanochannels of $\mathbf{2} \mathbf{b}$. In the ${ }_{25}$ ESR spectra, intense signals assigned to the propagating radicals of both PSt and PMMA were observed, and the maximum radical concentrations of PSt and PMMA in $\mathbf{2 b}$ reached 2.6 and $0.48 \mathrm{mmol} \mathrm{kg}^{-1}$, respectively, which are much higher than those detected in conventional solution 30 polymerizations $\left(10^{-4}-10^{-5} \mathrm{mmol} \mathrm{kg}{ }^{-1}\right)$. It should also be noted that the ESR signal of PSt did not disappear over three weeks, even at $70{ }^{\circ} \mathrm{C}$. These results clearly indicate stabilization of the growing radicals, caused by efficient suppression of termination reactions in the nanochannels.

${ }_{35}$ Consequently, smaller polydispersities $\left(M_{\mathrm{w}} / M_{\mathrm{n}}\right)$ of the polymers were obtained in the polymerizations using $\mathbf{1}$ and $\mathbf{2}$, compared with those of corresponding bulk-synthesized polymers (Fig. 3a). ${ }^{12,14}$ The molecular weight distributions of PSt and PMMA were found to become narrower with a ${ }_{40}$ decrease in the host channel size, and eventually a value for $M_{\mathrm{w}} / M_{\mathrm{n}}$ of 1.5 was attained in the polymerization of St using 2c. ${ }^{14}$

Unlike St and MMA, the propagating radical of poly(vinyl acetate) (PVAc) shows intractably high reactivity and less (a)

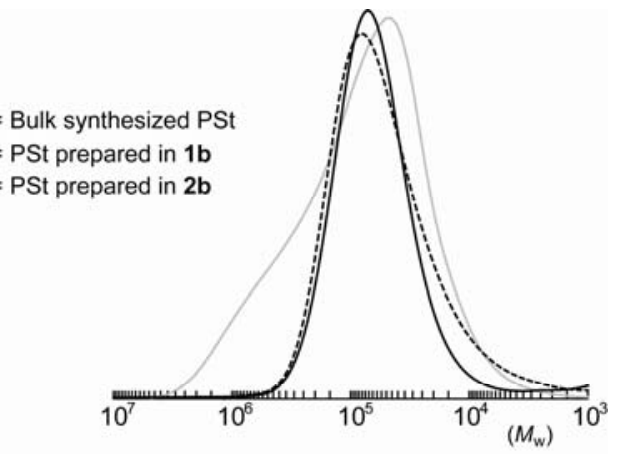

(b)

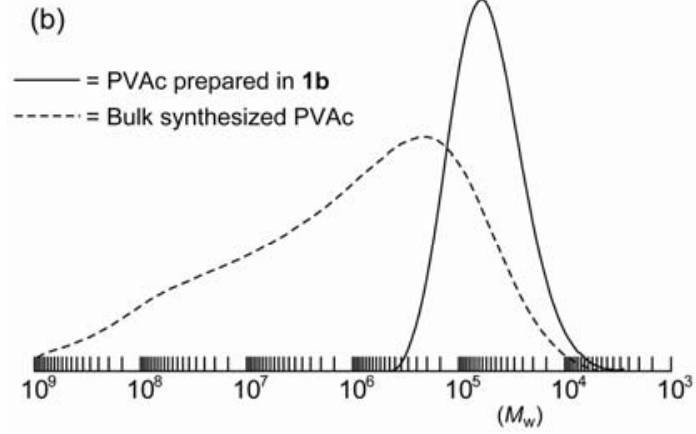

Fig. 3 GPC profiles of (a) PSt and (b) PVAc prepared in PCPs or bulk.

45 stability because of the lack of a conjugated substituent in the vinyl acetate (VAc) monomer. Thus, unfavorable chain transfer and termination reactions are particularly observed in the polymerization process of VAc, and the controlled radical polymerization of VAc has been very difficult to achieve. ${ }^{16}$ 50 However, PVAc obtained from the nanochannels showed small $M_{\mathrm{w}} / M_{\mathrm{n}}$ values (e.g., $M_{\mathrm{w}} / M_{\mathrm{n}}$ for PVAc prepared in $\mathbf{1 b}$ was 1.7), while PVAc prepared under bulk conditions showed an extremely large polydispersity $\left(M_{\mathrm{w}} / M_{\mathrm{n}}>20\right)$ (Fig. 3b). ${ }^{14}$ This demonstrates the narrow nanochannel effect of PCPs on ${ }_{55}$ suppressing side reactions in the VAc polymerization process.

\subsection{Control of stereoregularity}

The structural control of vinyl polymers to fabricate a desired stereoregularity (tacticity) is very important, because the tacticity of the polymers strongly affects the polymer 60 properties. However, the control of stereochemistry in radical polymerization has been considered more difficult because of the lack of efficient methods for providing an asymmetric environment around the propagating radical species. Recent developments in this area have emerged from general methods, 65 in which the addition of polar solvents or Lewis acids to the reaction media leads to effective interactions with the polar substituents of the monomers and/or around the propagating radical species to induce the stereospecific chain growth. ${ }^{10,11}$

In contrast to the through-bond interactions observed in 70 solution systems, a specific nanochannel effect on the polymer stereoregularity has been reported in polymerizations using $\mathbf{1}$ and 2, where through-space interactions play very important roles for controlling the stereoregularity. ${ }^{14}$ In this system, radical polymerization of vinyl monomers (St, MMA, and $75 \mathrm{VAc}$ ) has led to an increase in isotacticity in the resulting polymers. For example, the tacticity of PMMA strongly 

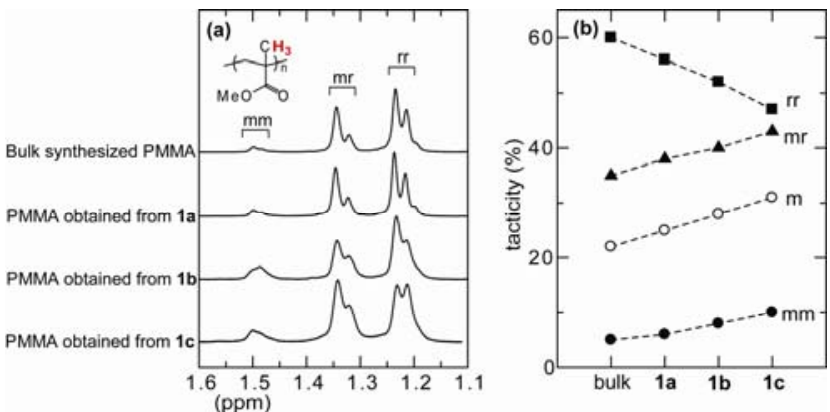

Fig. 4 (a) ${ }^{1} \mathrm{H}$ NMR spectra of PMMA obtained from the nanochannels of 1 and the bulk condition in nitrobenzene- $d_{5}$ at $110{ }^{\circ} \mathrm{C}$. (b) Plot of nanochannel effect on tacticity of PMMA.

depends on the pore size of the PCPs; eventually an increase of $9 \%$ in isotacticity (meso diad; $\mathrm{m}$ ) was achieved by using 1c compared with that obtained from the bulk polymerization system (Fig. 4). When the VAc monomer was polymerized in 5 the nanochannels of $\mathbf{2} \mathbf{b}$, the ratio of isotactic units in the PVAc structure clearly increased $(\mathrm{m}=55 \%)$ : this is the first example of an increase in the isotactic units in the polymerization of VAc. ${ }^{14}$ This stereospecific polymerization in PCPs can be explained by through-space interaction 10 between the pore walls and the monomers. Because an isotactic polymer requires a narrower conformational diameter (thickness) than the corresponding syndiotactic polymer, the polymerizations in nanochannels of the PCPs would result in increase in the less stereo-bulky isotactic unit.

15 The introduction of relatively weak functional groups, such as carbonyl, hydroxyl, nitro, amide, etc., into the nanochannels of PCPs could affect the monomer alignment, which may lead to precise control of stereoregularity of the resulting polymers. In particular, PCPs with either helical or 20 chiral structures on the pore surface are of intense interest. ${ }^{17}$ One can use such chiral channels to induce asymmetric polymerizations, which may give chiral or helical polymer conformations. Thus, utilization of the size-, shape-, and functionality-tunable pores of PCPs should be promising for 25 attaining stereoregulated radical polymerizations.

\subsection{Control of reaction sites}

Usually, multivinyl-substituted monomers such as divinylbenzenes (DVBs) are used as cross-linking agents in radical polymerization systems to produce functional gels, ion 30 exchange resins, adsorbents, molecularly imprinted polymers, supports for reagents in organic synthesis, and so on. In contrast to the conventional use of DVBs as cross-linkers, further progress hinges on the development of an efficient methodology for directing low-dimensional regulated radical polymerization, 35 which not only contributes to synthetic polymer chemistry, but also provides an attractive material that definitely shows different properties from those of the usual network polymers.

Interestingly, radical polymerization of DVBs in the onedimensional (1-D) channels of $\mathbf{1 b}$ and $\mathbf{2} \mathbf{b}$ gave a linear 40 polymer retaining pendant vinyl groups in all benzene rings (Fig. 5), which was confirmed by a solubility check and spectroscopic measurements, such as IR, UV-vis, and ${ }^{1} \mathrm{H}$ NMR spectra. ${ }^{13}$ In this system, effective entrapments of the propagating radical mediators in the 1-D nanochannels has led

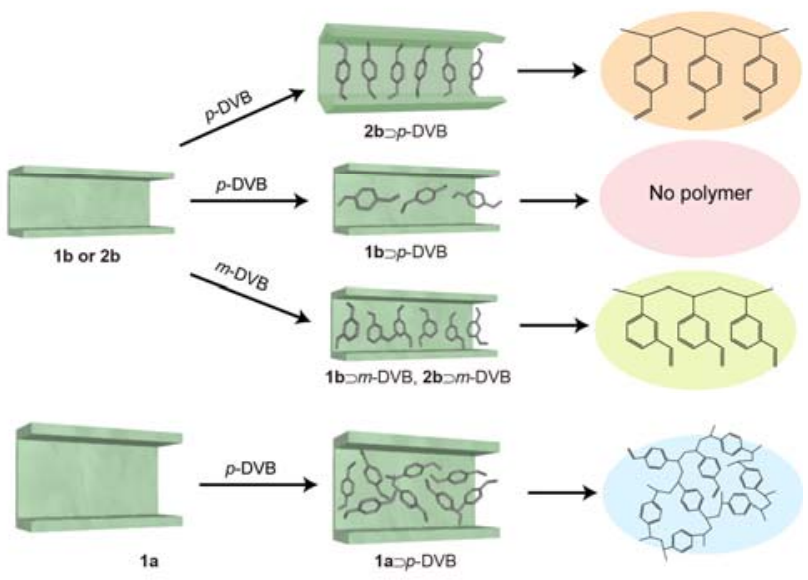

Fig. 5 Schematic images for inclusion and polymerization of DVBs in nanochannels of $\mathbf{1 a}, \mathbf{1} \mathbf{b}$, and $\mathbf{2} \mathbf{b}$.

45 to this linearly extended polymerization.

Further studies on the importance of channel characteristics for site-selective polymerization have shown that the framework flexibility of PCPs plays an important role in specific adsorption and polymerization of DVBs (Fig. 5). ${ }^{13}$ In 50 this study, introduction of 1,4-disubstituited DVB ( $p$-DVB; molecular dimension $=8.5 \times 4.4 \AA$ ) in the channels of $\mathbf{2 b}$ induces a lattice expansion compared with the original host; however, the isostructural copper compound $\mathbf{1 b}$ did not show such structural changes. In addition, the adsorbed amount of 55 the monomer in $\mathbf{1 b}$ was much smaller than that in $\mathbf{2 b}$. Consequently, polymer product was only obtained in the nanochannels of $\mathbf{2} \mathbf{b}$; in contrast, no trace of polymer was observed by using 1b. Thus, the host flexibility of $\mathbf{2 a}$ is strongly associated with adsorption of the monomer by 60 expanding the pore structure suited for adjacent arrangement of the monomer, resulting in the successful selective polymerization; although the robust isostructural copper compound $\mathbf{1 b}$ did not perform well for this polymerization.

To elucidate the importance of the channel size for this 65 topotactic polymerization, polymerization of $p$-DVB in the 1a framework with large open channels (pore size $=10.8 \times 10.8$ $\AA^{2}$ ) was studied. ${ }^{13}$ In this case, the polymerization proceeded with high conversion (63\%); however, the obtained polymeric product isolated from the channels was insoluble in any 70 solvents, showing that semibranching cross-linked structures were formed in this large channel. This fact indicates that the design of a channel size suitable for single molecule accommodation is also a key factor for the selective linear polymerization (Fig. 5).

75 Because of the nonconjugated nature of the propagating radical of PVAc, unfavorable side reactions (back bitings) are particularly observed in the radical polymerization process of VAc; thus, the resultant PVAc usually has many branching structures. However, polymerization of VAc in the 80 nanochannels of PCPs has produced less-branched forms of PVAc, which is ascribed to the constrained chain growth in the narrow 1-D nanochannels. ${ }^{14}$ 


\subsection{Control of copolymer composition and sequence}

The nucleic acid-templated polymerizations of DNA, RNA, and proteins are essential processes of living systems. Templatedirected polymerization mediates the translation of genetic 5 information into functional macromolecules by attaining precision control of the copolymer sequence. Thus, control of composition and sequence in artificial copolymerization systems is a great challenge. ${ }^{18}$ From this point of view, radical copolymerizations of vinyl monomers, such as St, MMA, and ${ }_{10} \mathrm{VAc}$, were examined in the nanochannels of $\mathbf{1} \mathbf{b}{ }^{15}$ In this system, it is generally seen that compositions of St in the copolymers prepared in the host are lower than those obtained from the corresponding free radical systems (Fig. 6). This change of monomer reactivity in the nanochannels was explained by the 15 molecular size and electronic properties of the monomer. The interaction potential between the $\mathrm{St}$ molecule and the nanochannel of $\mathbf{1 b}$ seems large because the molecular size of St $(6.8 \times 4.4 \AA)$ is similar to that of the pore, and an effective $\pi-\pi$ interaction between the monomer and the pore is also expected.

20 This results in a decrease in the diffusion speed of St in the channel, compared with those of MMA $(5.9 \times 4.1 \AA)$ and VAc $(5.5 \times 4.0 \AA)$. In addition, the reactivity of the large St toward polymer chain terminals may be more restricted in the nanochannels. Thus, these results suggest a possibility that 25 copolymerization utilizing PCP nanochannels could have a significant influence on the composition and sequence in the copolymer structures, by efficient through-space inductions.
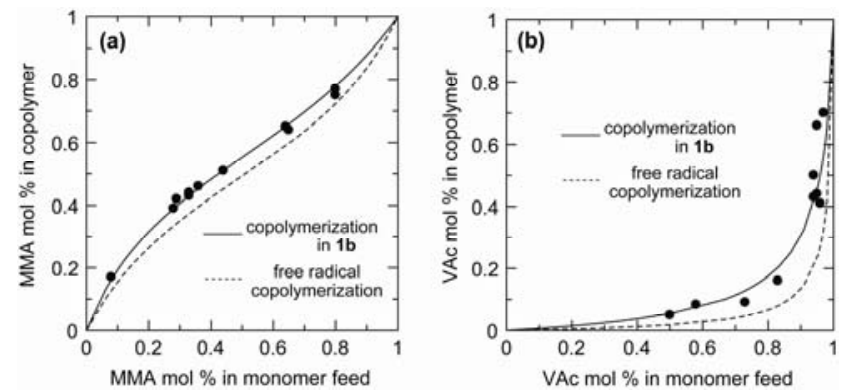

Fig. 6 Copolymer composition curves obtained from copolymerizations of (a) St and MMA and (b) St and VAc in the nanochannels of $\mathbf{1 b}$.

\section{Channel-promoted polymerizations in PCPs}

There is a growing interest in the study of PCPs with 30 functionalized pore surfaces. The versatile pore features of PCPs, such as redox activity, Lewis acidity, basicity, and chirality, can be tailored by regularly arranged functional organic ligands and metal ions. ${ }^{19}$ Thus, PCPs bearing active sites in/on nanochannels have been envisioned for useful catalytic applications in organic 35 and polymer syntheses. In particular, an attractive target in polymer synthesis is the utilization of active sites for fabricating $\pi$-conjugated polymers that show interesting optical and electrical properties. Precisely aligned PCP nanopores filled with $\pi$ conjugated polymers should be a promising new class of host-

40 guest nanostructures for fundamental investigations on lowdimensional properties of these polymers in the isolated state and for applications in the creation of nanosized molecule-based devices.

\subsection{Ligand-promoted polymerization}

${ }_{45}$ Much attention has been directed to the physical functions of poly(substituted acetylenes) for a wide range of applications, such as conducting materials, nonlinear optics, and polymer sensors. $^{20}$ The controlled polymerization of substituted acetylenes by using several types of catalysts has been 50 investigated with the aim of controlling the stereostructure (the cis and trans chain sequence) and decreasing the formation of cyclic trimer by-products (substituted benzenes) because the characteristic properties of poly(substituted acetylenes), such as conjugation length, supra-structures, and processability, can be 55 precisely adjusted by changing the substituents and stereoregularity.

Substituted acetylenes were polymerized in 1-D nanochannels of the Coordination Pillared-Layer (CPL) compounds $\left[\mathrm{Cu}_{2}(\mathrm{pzdc})_{2}(\mathrm{~L})\right]_{\mathrm{n}}$ (pzdc = pyrazine-2,3-dicarboxylate, $\mathrm{L}=$ pillar 60 ligands) with basic carboxylate oxygen atoms as catalytic interaction sites (Fig. 7a). ${ }^{21}$ For example, acidic methyl propiolate (MP) is encapsulated in the nanochannels of $\left[\mathrm{Cu}_{2}(\text { pzdc })_{2}\left(4,4^{\prime} \text {-bipyridine }\right)\right]_{\mathrm{n}}(\mathbf{C P L}-2$; channel size $=8.2 \times 6.0$ $\AA^{2}$ ) and interacts with the basic oxygen atoms on the pore surface. ${ }_{65}$ As a consequence of the acid-base interaction, C- $\mathrm{H}$ bond dissociation of MP produces a reactive acetylide species that initiates polymerization, which was characterized by IR measurements. Molecular-mechanics structure optimization also reproduced a specific hydrogen-bonding interaction between the 70 MP monomer and the carboxylate moiety of the host CPL-2 (Fig. $7 b){ }^{22}$ The acetylenic hydrogen atom and the oxygen atoms are close enough for efficient hydrogen bonding (2.8 and $2.9 \AA$ ). In addition, IR and adsorption spectra of polyMP extracted from the nanochannels of CPL-2 indicated the dominance of the highly 75 conjugated trans form of polyMP. The narrow nanochannel structure might direct the selectivity toward a polymerization with trans addition because of the prohibitive steric demand for the formation of trisubstituted benzenes and cis polymers.

In a control experiment, MP was treated with sodium benzoate 80 as a discrete model catalyst at room temperature and the mixture was left for one month, resulting in only a trace amount of product. An increase in the reaction temperature to $70{ }^{\circ} \mathrm{C}$ resulted in a small quantity of yellow oily mixture $(<5 \%)$ of cyclic trisubstituted benzenes as the major products and less-conjugated ${ }_{85}$ polyMP with cis geometry as a minor product, which contrasts with the result obtained in the nanochannels of CPL-2. The polymerization system with PCP is of considerable interest because of several significant features, such as a dramatic acceleration of the polymerization, no production of trisubstituted 90 benzenes as byproducts, and trans-selective polymerization. (a)

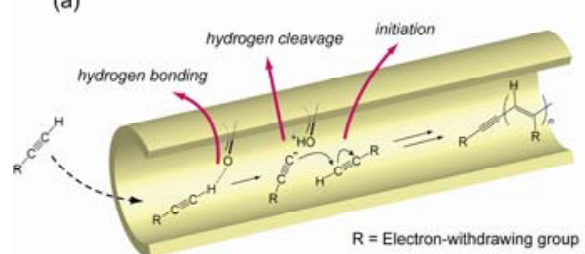

(b)

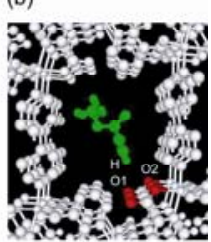

Fig. 7 (a) Polymerization mechanism of the ligand-catalytic polymerization of acidic acetylenes in CPLs. (b) Optimized structure of MP incorporated in a nanochannel of CPL-2. 


\subsection{Metal-promoted polymerization}

A wide range of $\pi$-conjugated polymers with important electrical and optical properties can be prepared by oxidative polymerization. For example, typical conducting polymers such 5 as polypyrrole (PPy), polythiophene, polyaniline, and their derivatives are synthesized via oxidative polymerization. Therefore, it is important to develop a method for oxidatively polymerizing monomers in PCPs for the preparation of functional nanohybrids between coordination and conducting organic ${ }_{10}$ polymers.

PCPs with redox-active metal ions might be capable of providing controlled reactions and orientations for oxidized/reduced products within the resulting crystalline composites. However, only a few reports on the use of PCPs as ${ }_{15}$ such significant redox reaction fields have been reported, ${ }^{23}$ because in many cases, decomposition of the host framework is involved during the redox reaction. Recent work on the polymerization of pyrrole (Py) within a redox-active twodimensional (2-D) layered framework, $20\left[\mathrm{Ni}(\mathrm{dmen})_{2}\right]_{2}\left[\mathrm{Fe}^{\mathrm{IIII}}(\mathrm{CN})_{6}\right]\left(\mathrm{PhBSO}_{3}\right) \quad(3 ;$ dmen $=1,1-$ dimethylethylenediamine; $\mathrm{PhBSO}_{3}{ }^{-}=p$-phenylbenzenesulfonate), showed that $\left[\mathrm{Fe}^{\mathrm{III}}(\mathrm{CN})_{6}\right]$ units of $\mathbf{3}$ oxidized and polymerized Py monomers within the host framework, which was confirmed by IR and magnetic susceptibility measurements (Fig. 8). ${ }^{24}$ In 25 addition, XRPD measurements indicated that the resulting PPy was intercalated between the 2-D sheets of $\mathbf{3}$ and that a layer-bylayer-type crystalline nanocomposite was synthesized.

Template synthesis has the potential to control the structure and morphology of products obtained after removal of the host matrix. 30 Removal of the host framework in EDTA solution allowed isolation of the intercalated PPy as an insoluble black precipitate. The controlled morphogenesis of the isolated polymer objects is evident from the SEM images. In contrast to the granular morphology of the bulk PPy prepared by oxidation of Py with ${ }_{35} \mathrm{~K}_{3}\left[\mathrm{Fe}^{\mathrm{III}}(\mathrm{CN})_{6}\right]$ in water, it is obvious that the morphology of the isolated PPy is finely platy. An XRPD measurement indicated that the bulk PPy was found to be an amorphous compound; in contrast, the XRPD profile of the isolated PPy showed the accumulation of aromatic planes of PPy. In addition, the 40 obtained PPy microsheet object showed anisotropic conductivity; the conductivity parallel to the sheet was 20 times higher than that along the direction perpendicular to the sheet. Therefore, in this system, the crystalline template $\mathbf{3}$ successfully directed the
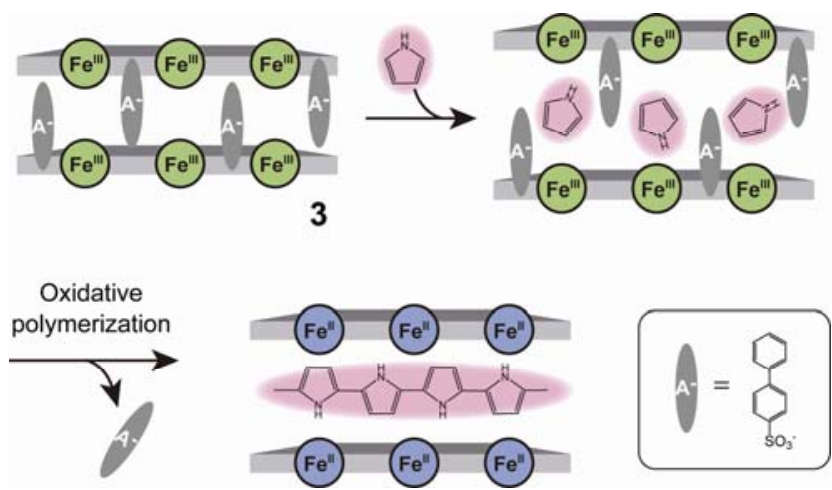

Fig. 8 Schematic illustration of the intercalation of Py and the oxidative polymerization of Py by the Fe(III) ions in the host layers. well-structured and 2-D oriented organization of PPy assembly at ${ }_{45}$ the molecular level. ${ }^{24}$ These results indicate that PCPs can be used as templates for controlling the orientation and organization of polymer architectures at one-, two-, and three-dimensional (3D) levels.

\section{Confined polymers and their properties in PCPs}

50 Polymers confined in nanospaces will have distinctly different properties from those in the bulk state because of the formation of specific molecular assemblies and conformations. In particular, polymer inclusion in crystalline microporous hosts (pore size $<2$ $\mathrm{nm}$ ) with ordered and well-defined nanochannel structures is 55 attracting much attention. This is largely because this approach can prevent the entanglement of polymer chains and provide extended chains in restricted spaces, in contrast to amorphous bulk polymer systems and polymers in solution. In this field, microporous organic hosts such as ureas, perhydrotriphenylene, 60 cyclotriphosphazenes, cholic acids, and cyclodextrins play dominant roles. ${ }^{25}$ However, these organic hosts exploit only hydrogen bonding or weak van der Waals interactions and generally produce narrow channels (ca. 4-6 A) that often prevent the incorporation of polymer chains with bulky side chains, such 65 as PSt, polyacrylates, and polymethacrylates.

For the inclusion compounds of polymers with PCPs, it is possible to investigate the properties of confined single polymer chains. The usefulness of this method was demonstrated by the recent study of the conformation and dynamics of single PSt ${ }_{70}$ chains confined in $\mathbf{2} \mathbf{b} .^{26}$ Differential scanning calorimetry of bulk PSt showed a $T_{\mathrm{g}}$ at $105{ }^{\circ} \mathrm{C}$; in contrast, the PSt in the channels of $\mathbf{2 b}$ did not show such a transition. The suppression of the $T_{\mathrm{g}}$ suggests that the chain assembly of PSt was considerably changed in the host-guest adduct. Interestingly, 75 single PSt chains in regular 1-D nanochannels afforded unique molecular dynamics that are quite different from those observed in the bulk state. Solid-state ${ }^{2} \mathrm{H}$ NMR measurement for a bulk [D $\left.\mathrm{D}_{5}\right]$ PSt sample and a nanocomposite of $\left[\mathrm{D}_{5}\right] \mathrm{PSt}$ with $\mathbf{2 b}$ indicated that bulk $\left[\mathrm{D}_{5}\right] \mathrm{PSt}$ provides a wide motional distribution 80 that originates from the heterogeneous local environment of the phenyl rings; in contrast, PSt in $\mathbf{2 b}$ represents a quasi-single-type phenyl flipping motion, indicating homogeneous side-chain mobility in the nanochannel. In addition, the activation energy for the phenyl flip of the PSt chain in $\mathbf{2} \mathbf{b}$ was significantly lower ${ }_{85}\left(E_{\mathrm{a}}=8.8 \mathrm{~kJ} \mathrm{~mol}^{-1}\right)$ than that of bulk PSt because of the lower steric hindrance of the phenyl rings of the single PSt chain in the nanochannel.

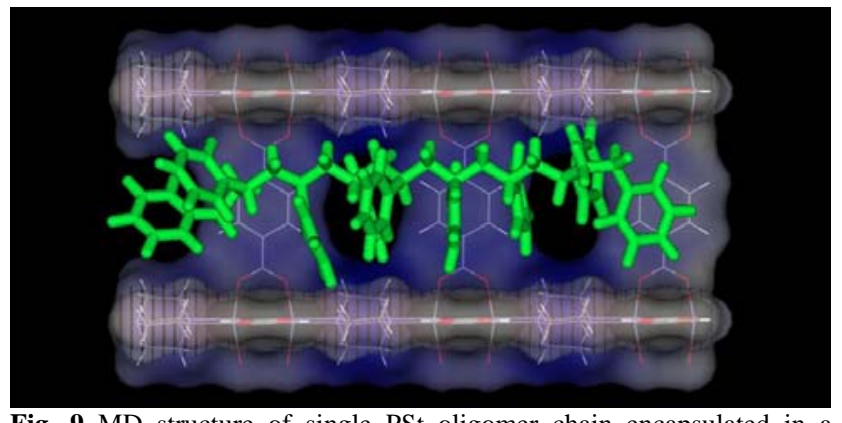

Fig. 9 MD structure of single PSt oligomer chain encapsulated in a nanochannel of $\mathbf{2 b}$. 
The chain conformations of PSt oligomers in the 1-D channel of 2b, simulated using the molecular dynamics (MD) method, agree with the unique molecular motion observed in the NMR measurements. In the MD simulation, the introduction of a 5 higher molecular weight PSt into the nanochannel led to a highly extended linear conformation of the single PSt chain (Fig. 9), which contrasts significantly with the random chain-entangled structures that essentially occur in the bulk state. These results indicate the possibility of PCP nanovessels for providing

10 significant insights into polymer-confinement effects, as well as inherent properties of single polymer chains, because PCPs can supply tailor-made nanopores with a variety of pore sizes and environments.

\section{Fabrication of inorganic polymers in/at PCPs}

15 Metals and metal oxides, which can be regarded as "inorganic polymers", display many interesting optical, electronic, chemical, and catalytic properties in the nanometer-size regime. ${ }^{27}$ Such nanoscale materials have potential applications in developing biological nanosensors and optoelectronic nanodevices. Much

20 research activity has been seen in recent years in the area of hostguest nanocomposites based on nanosized inorganic polymers and various host matrices because of specific size-dependent properties and host-guest synergistic functions. One promising idea is the preparation of inorganic polymers in the pores of PCPs,

25 which would lead to potential applications ranging from fundamental physical studies to trace molecular detection and high-surface-area catalysis.

\subsection{Metal nanoparticles in/at PCPs}

Recently, studies on the formation of metal nanoparticles so utilizing PCP frameworks have been reported by several groups. The works of Suh et al. have shown that redox-active $\mathrm{Ni}^{\mathrm{II}}$-cyclam sites in PCPs play an important role in reducing metal ions to form small metal nanoparticles. ${ }^{28-30}$ Interestingly, immersion of [ $\mathrm{Ni}\left(\mathrm{C}_{10} \mathrm{H}_{26} \mathrm{~N}_{6}\right)$ (BPDC)] (4; BPDC ${ }_{35}=$ biphenyl-4,4'-dicarboxylate) in methanolic solution of $\mathrm{AgNO}_{3}$ afforded silver nanoparticles with a diameter of $3 \mathrm{~nm}$ (Fig. 10). ${ }^{28}$ ESR and energy-dispersive X-ray measurements suggested that a redox reaction between $\mathrm{Ag}^{+}$and $\mathrm{Ni}^{2+}$ in the host is the key to forming silver nanoparticles. However, the 40 size of the nanoparticles seems larger than the pore size of $\mathbf{4}$. In addition, the XRPD patterns of $\mathbf{4}$ changed completely and became much weaker after the long-time reaction, showing that the original host framework was destroyed. Thus, they have developed this method using a 2-D (layered) PCP ${ }_{45}\left[\mathrm{Ni}_{2}(\text { cyclam })_{2}(\mathrm{BPTC})\right]_{n} \quad\left(\mathrm{BPTC}=1,1^{\prime}\right.$-biphenyl-2,2',6,6'tetracarboxylate $)^{29}$ or a PCP with interpenetrating diamondoid structure $\quad\left[\mathrm{Ni}_{2}(\mathrm{cyclam})_{2}(\mathrm{MTB})\right]_{n} \quad$ (MTB = methanetetrabenzoate $)^{30}$. Treatment of those redox-active porous solids with solutions of various metal ions produced 50 metal nanoparticles, such as silver ( $3.7 \pm 0.4 \mathrm{~nm})$, gold $(2 \mathrm{~nm})$, and palladium $(2.0 \pm 0.6 \mathrm{~nm})$, with maintenance of the host framework. Their proposed mechanism for the formation of the nanoparticles involves metal ions reacting with $\mathrm{Ni}$ species within the host channels to form metal atoms, which diffuse to 55 the surface of the solids to grow into nanoparticles.

Chemical vapor deposition (CVD) of organometallic

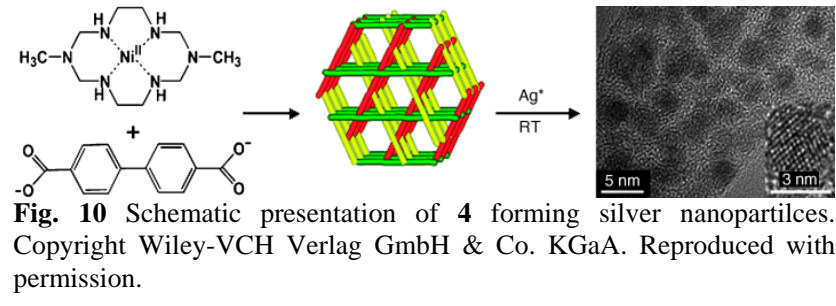

precursors into frameworks of PCPs offers a promising perspective for the formation of metal nanoparticles. ${ }^{31-33}$ For example, Fischer et al. have reported that loading of the $\mathrm{Pd}$ 60 precursor $\left[\left(\eta^{5}-\mathrm{C}_{5} \mathrm{H}_{5}\right) \mathrm{Pd}\left(\eta^{3}-\mathrm{C}_{3} \mathrm{H}_{5}\right)\right]$ in $\left[\mathrm{Zn}_{4} \mathrm{O}(\mathrm{BDC})_{3}\right]_{n}$ (MOF5; $\mathrm{BDC}=1$,4-benzenedicarboxylate) by CVD afforded $\mathrm{Pd}$ nanoparticles with dimensions of $1.4 \pm 0.1 \mathrm{~nm}$ after treatment of the inclusion compound with $\mathrm{H}_{2}$ gas. ${ }^{31}$ This method can be applied to produce a variety of metal nanoparticles, such as 65 copper (1-4 nm), gold (5-20 nm), and ruthenium (1.5-1.7 $\mathrm{nm}),{ }^{31-33}$ because many volatile organometallic precursors can be loaded by CVD. Even though the diffraction peaks of MOF-5 in the XRD profiles became weaker after $\mathrm{H}_{2}$ treatment, the resultant metal nanoparticles are likely to 70 remain situated inside the porous structures, except for the case of gold. Alternatively, photo-assisted thermolysis of MOF-5 loaded with $\mathrm{Cu}$ precursors could also form nanoparticles in the framework. ${ }^{33}$ It is noteworthy that these metal nanoparticles in MOF-5 showed catalytic activities, 75 such as hydrogenation of olefins, methanol synthesis, and alcohol oxidation. ${ }^{31-34}$ A recent breakthrough showed the selective growth of patterned thin films of MOF-5 following loading with $\left[\left(\eta^{5}-\mathrm{C}_{5} \mathrm{H}_{5}\right) \mathrm{Pd}\left(\eta^{3}-\mathrm{C}_{3} \mathrm{H}_{5}\right)\right]$, which acted as a precursor for the deposition of palladium nanoparticles inside 80 the cavities of the MOF-5 material. ${ }^{35}$ It is currently possible to perform simultaneous characterizations of the MOF-5 framework and the loaded metal particles by using advanced TEM techniques. ${ }^{36}$

Very recently, another interesting method for the 85 encapsulation of metal particles into a PCP has been reported. The formation of metal nanoparticles comprised the incorporation of anionic metal precursors, such as $\left[\mathrm{PdCl}_{4}\right]^{2-}$, $\left[\mathrm{PtCl}_{4}\right]^{2-}$, and $\left[\mathrm{AuCl}_{4}\right]^{--}$, into the porous framework of aminegrafted chromium tetephthalate (MIL-101) by anionic 90 exchange, followed by reduction with $\mathrm{NaBH}_{4}$ at low temperature. ${ }^{37}$ TEM measurement showed successful encapsulation with the detection of fine nanoparticles in the range of 2-4 nm, in agreement with the cage diameter, although some nanoparticles still remained outside the pores. 95 It is noteworthy that materials loaded with Pd nanoparticles on MIL-101 have very high activity during the Heck reaction.

\subsection{Metal oxides in PCPs}

Metal oxides materials are attractive in applications such as insulators, ceramics, and catalyst supports. The sol-gel 100 method for metal oxide synthesis has become a developing technique for the preparation of a variety of nanostructured materials and functional nanocomposites. This process is defined as the polymerization of metal oxides precursors to produce a sol, which eventually grows into a gel network in 105 mild ambient conditions. Silica $\left(\mathrm{SiO}_{2}\right)$ is one of the most 


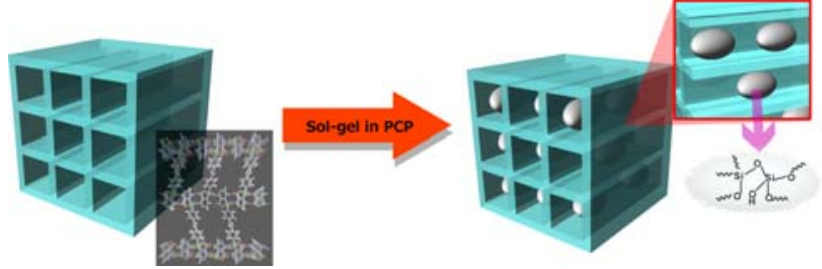

Fig. 11 Schematic image for sol-gel synthesis of low-dimensional silica in nanocahnnels of CPL-5.

useful metal oxide materials because of its transparency, stability, dielectric properties, and hydrophilicity. However, detailed properties of silica materials of $\sim 1 \mathrm{~nm}$ are still not clear because of the difficulty of precision control of silica 5 growth in this region (where the size of the $\mathrm{SiO}_{4}$ tetrahedron = $2.6 \AA)$. Very recently, the fabrication of silica in 1-D subnanochannels of $\left[\mathrm{Cu}_{2} \text { (pzdc) }\right)_{2}\{1,2$-di(pyridyl)ethylene $\left.\}\right]_{\mathrm{n}}$ (CPL-5; channel size $\left.=10.3 \times 6.0 \AA^{2}\right)$ using a sol-gel reaction of tetramethoxysilane has been reported (Fig. 11). ${ }^{38}$ In this 10 system, the sol-gel condensation successfully proceeded only inside the channels, without destruction of the host framework or deposition of silica particles outside the pores, as demonstrated by XRD, $\mathrm{N}_{2}$ adsorption, laser light diffraction, and electron microscope measurements. ${ }^{29} \mathrm{Si}$ magic angle 15 spinning NMR measurements clearly show that, unlike bulksynthesis systems, the growth of silica was effectively constrained, and the resultant low-dimensional silica was stably restricted in the channels of CPL-5. Moreover, study of the crystal phase transformation of silica in CPL-5 20 revealed a drastic decrease of its crystallization temperature as a result of its miniaturized size. Because an enormous range of metal oxide materials can be prepared by the sol-gel process, this methodology will contribute to new preparation systems not only for subnanosized metal oxides, but also for 25 functional nanohybrids between PCPs and metal oxides.

Recently, the loading of MOF-5 with $\mathrm{Cu}_{2} \mathrm{O}$ and $\mathrm{ZnO}$ nanoparticles has been attained. ${ }^{33}$ In the case of $\mathrm{Cu}_{2} \mathrm{O}$, asprepared $\mathrm{Cu}$ nanoparticles embedded in MOF-5 were surface oxidized by $\mathrm{N}_{2} \mathrm{O}$ treatment, resulting in a $\mathrm{Cu}_{2} \mathrm{O} / \mathrm{Cu}$ at $\mathbf{M O F}-5$ 30 material. Interestingly, reduction of this material with $\mathrm{H}_{2}$ gas reversibly formed $\mathrm{Cu}$ nanoparticles again. Preparation of $\mathrm{ZnO}$ nanoparticles $(<4 \mathrm{~nm}$ ) in MOF-5 was performed by loadimg MOF-5 with $\mathrm{ZnEt}_{2}$ followed by hydrolysis or dry oxidation. Doubly-loaded $\mathrm{Cu} / \mathrm{ZnO}$ at MOF-5 materials were also 35 prepared, which showed significant catalytic activity in methanol synthesis from a $\mathrm{CO} / \mathrm{CO}_{2} / \mathrm{H}_{2}$ gas mixture. ${ }^{33}$ This result suggests a superior interfacial contact between $\mathrm{Cu}$ and the $\mathrm{ZnO}$ nanophase in the MOF-5 channels.

\section{Conclusion}

40 In this review, we have given a brief survey of work, drawn primarily from the authors' group, directed toward understanding the synthesis and properties of polymers in PCPs. The nanochannels of PCPs have proven to be desirable fields not only for precision synthesis of polymer materials, 45 but also for exploring specific properties of polymer confinement. The ability to control the framework structures of PCPs makes us confident that polymer synthesis at our desire is no longer a dream for the future. In addition, construction of nanohybrids between PCPs and polymers will 50 provide unprecedented material platforms to accomplish many nanoscale functions. Considering the characteristic features of PCPs, there are and remain many fundamental and fascinating issues about polymer chemistry that can be performed in the nanochannels of PCPs, as discussed below.

55 Nano-information transcription polymerizations: One can program nanosized information, such as channel length and dimensionality, surface functionality, and pore size and shape, into the frameworks of PCPs, because of the infinite combinations between the metal ions and organic ligands. 60 Incorporation of monomers into the host framework can allow their programmed arrangement to be polymerized. After the polymerization, the host PCPs can be removed in mild ambient conditions, thus resulting in the isolation of polymer materials with controlled structures directly related to the 65 nano-information of PCPs. This methodology contributes not only to multiple controls of polymer primary structures, such as molecular weight, stereostructure, reaction position, and monomer composition and sequence, but also to a dimension-
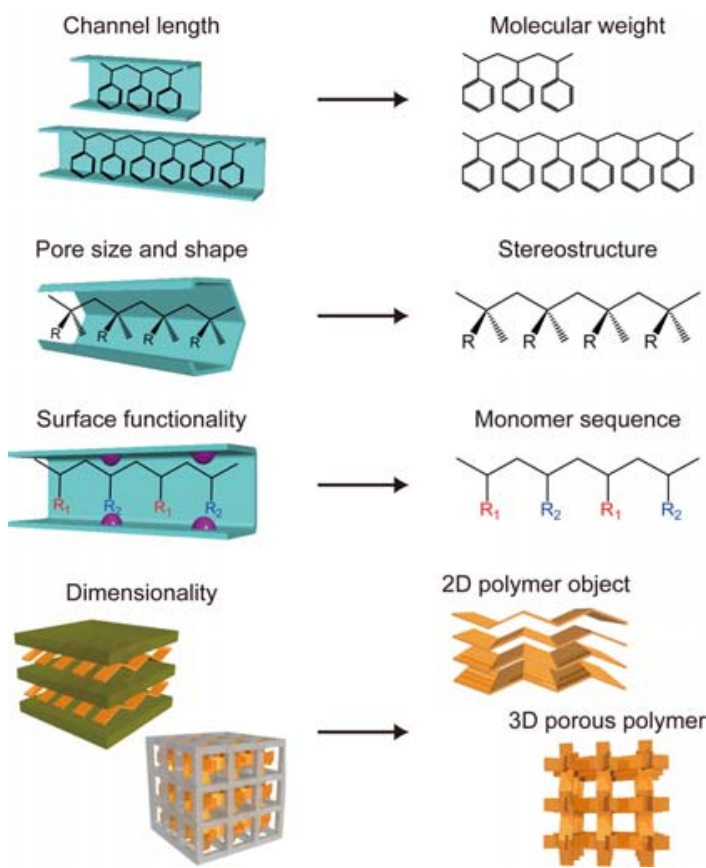

Stereostructure

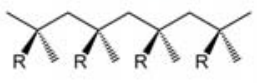

Monomer sequence

Fig. 12 Multiple controls of polymer structures by nano-information transcription polymerizations.

(2) Environment

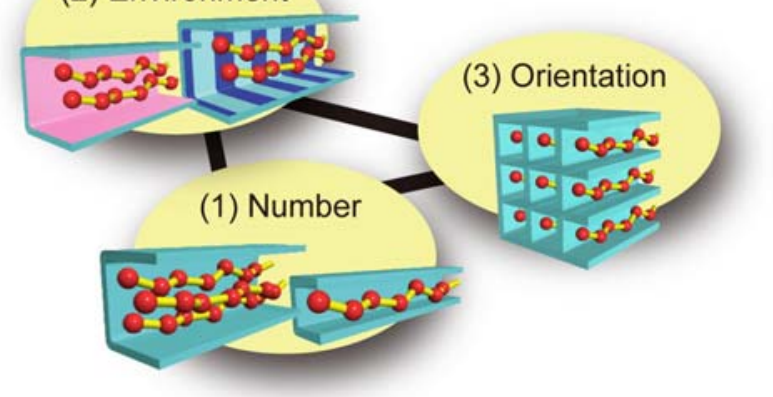

Fig. 13 Precise controls of polymer assemblies can be attained in designable nanochannels of PCPs. 
controlled polymerization in layered or 3-D intersecting channels of PCPs to produce anisotropic 2-D polymer objects or porous polymer materials (Fig. 12).

Chain-confinement chemistry: The regulated and tailor5 made pore characteristics of PCPs are also of key importance for achieving unique polymer encapsulation systems. We are now able to control precisely the following features within the designable nanochannels of PCPs: (1) the number of polymer chains, (2) the environments for polymers, and (3) the chain 10 orientations (Fig. 13). This concept leads to quantitative analysis of low-dimensional properties of polymer assemblies (single, double, triple, $\cdots$ chains) in specific porous environments and orientations, and to the preparation of a new class of materials based on PCP-polymer hybridization.

15 Understanding and controllig the polymer properties within the $2 \mathrm{~nm}$ region will give valuable information for their future applications in nanosized molecular-based devices.

\section{Notes and references}

${ }^{a}$ Department of Synthetic Chemistry and Biological Chemistry, Graduate 20 School of Engineering, Kyoto University, Katsura, Nishikyo-ku, Kyoto 615-8510, Japan. Fax: +81-75-383-2732; Tel: +81-75-383-2733; Email: kitagawa@sbchem.kyoto-u.ac.jp

${ }^{b}$ PRESTO, Japan Science and Technology Agency (JST), Honcho Kawaguchi, Saitama 332-0012, Japan.

$25{ }^{c}$ Institute for Integrated Cell-Material Sciences (iCeMS), Kyoto

University, Yoshida, Sakyo-ku, Kyoto 606-8501, Japan.

1 Polymerization in Organized Media, ed. C. M. Paleos, Gordon \& Breach: New York, 1992; K. Sada, M. Takeuchi, N. Fujita, M. Numata and S. Shinkai, Chem. Soc. Rev., 2007, 36, 415.

2 T. Uemura, S. Horike and S. Kitagawa, Chem. Asian J., 2006, 1, 36.

3 K. Tajima and T. Aida, Chem. Commun., 2000, 2399; M. Farina, G. Di Silvestro and P. Sozzani, In Comprehensive Supramolecular Chemistry, Vol. 10; ed. D. Reinhoudt, Pergamon: Oxford, 1996, pp 371; M. Miyata, In Comprehensive Supramolecular Chemistry, Vol. 10; ed. D. Reinhoudt, Pergamon: Oxford, 1996; pp 557; A Matsumoto, Polym. J., 2003, 35, 93; K. Moller and T. Bein, Chem. Mater., 1998, 10, 2950; S. A. Davis, M. Breulmann, K. H. Rhodes, B. Zhang and S. Mann, Chem. Mater., 2001, 13, 3218; K. J. C. van

40 Bommel, A. Friggeri and S. Shinkai, Angew. Chem. Int. Ed., 2003, 42, 980.

4 J. C. Hulteen and C. R. Martin, J. Mater Chem., 1997, 7, 1075; D. J. Cardin, Adv. Mater., 2002, 14, 553.

5 O. M. Yaghi, M. O’Keeffe, N. W. Ockwig, H. K. Chae, M. Eddaoud

45 and J. Kim, Nature, 2003, 423, 705; S. Kitagawa, R. Kitaura and S. i. Noro, Angew. Chem. Int. Ed., 2004, 43, 2334; G. Férey, Chem. Soc. Rev., 2008, 37, 191; C. J. Kepert, Chem. Commun., 2006, 695.

6 The Chemistry of Radical Polymerization, Second Edition, ed. G. Moad and D. H. Solomon, Elsevier: Oxford, 2006; Handbook of Radical Polymerization, ed. K. Matyjaszewski and T. P. Davis, Wiley-Interscience: Hoboken, 2002.

7 W. A. Braunecker and K. Matyjaszewski, Prog. Polym. Sci., 2007, 32, 93.

8 G. Moad, Y. K. Chong, A. Postma, E. Rizzardo and S. H. Thang, Polymer, 2005, 46, 8458.

9 M. Kamigaito, T. Ando and M. Sawamoto, Chem. Rec., 2004, 4, 159.

10 M. Kamigaito and K. Satoh, Macromolecules, 2008, 41, 269.

11 S. Habaue and Y. Okamoto, Chem. Rec., 2001, 1, 46.

12 T. Uemura, K. Kitagawa, S. Horike, T. Kawamura, S. Kitagawa, M. Mizuno and K. Endo, Chem. Commun., 2005, 5968

13 T. Uemura, D. Hiramatsu, Y. Kubota, M. Takata and S. Kitagawa, Angew. Chem. Int. Ed., 2007, 46, 4987.

14 T. Uemura, Y. Ono, K. Kitagawa and S. Kitagawa, Macromolecules, 2008, 41, 87.

6515 T. Uemura, Y. Ono and S. Kitagawa, Chem. Lett., 2008, 37, 616.
16 S. Yamago, B. Ray. K. Iida, J.-i. Yoshida, T. Tada, K. Yoshizawa and T. Fukuda, J. Am. Chem. Soc., 2004, 126, 13908; K. Koumura K. Satoh, M. Kamigaito and Y. Okamoto, Macromolecules, 2006, 39, 4054; A. Debuigne, J. R. Caille and R. Jérôme, Macromolecules, 2005, 38, 5452.

17 D. Bradshaw, J. B. Claridge, E. J. Cussen, T. J. Prior and M. J. Rosseinsky, Acc Chem. Res., 2005, 38, 273; B. Kesanli and W. Lin Coord. Chem. Rev., 2003, 246, 305.

18 R. E. Kleiner, Y. Brudno, M. E. Birnbaum and D. R. Liu, J. Am. Chem. Soc., 2008, 130, 4646.

19 M. Higuchi, S. Horike and S. Kitagawa, Supramol. Chem., 2007, 19, 75; S. Kitagawa, S.-i. Noro and T. Nakamura, Chem. Commun., 2006, 701.

20 J. W. Y. Lam and B. Z. Tang, Acc. Chem. Res., 2005, 38, 745; K. J. S. Harrell and S. T. Nguyen, In Handbook of Advanced Electronic and Photonic Materials and Devices, Vol 8, Academic Press: San Diego, 2001; pp 131.

21 T. Uemura, R. Kitaura, Y. Ohta, M. Nagaoka and S. Kitagawa, Angew. Chem. Int. Ed., 2006, 45, 4112.

8522 Y. Ohta, H. Hitomi, M. Nagaoka, R. Matsuda, T. Uemura and S. Kitagawa, J. Am. Chem. Soc., 2008, under revision.

23 H. Choi and M. P. Suh, J. Am. Chem. Soc., 2004, 126, 15844; J. W. Han, and C. L. Hill, J. Am. Chem. Soc., 2007, 129, 15094.

24 N. Yanai, T. Uemura, M. Ohba, Y. Kadowaki, M. Maesato, M. Takenaka, S. Nishitsuji, H. Hasegawa and S. Kitagawa, Angew. Chem. Int. Ed., 2008, in press.

25 A. P. Primrose, M. Parves and H. R. Allcock, Macromolecules, 1997, 30, 670; J. Liu, P. A. Mirau and A. E. Tonelli, Prog. Polym. Sci., 2002, 27, 357; P. Sozzani, S. Bracco, A. Comotti and R. Simonutti,

$95 \quad$ Adv. Polym. Sci., 2005, 181, 153.

26 T. Uemura, S. Horike, K. Kitagawa, M. Mizuno, K. Endo, S. Bracco, A. Comotti, P. Sozzani, M. Nagaoka and S. Kitagawa, J. Am. Chem. Soc., 2008, 130, 6781.

27 Clusters and Colloids, ed. G. Schmid, VCH: Weinheim, 1994; H. Weller, Adv. Mater., 1993, 5, 88; P. V. Kamat, J. Phys. Chem. B, 2002, 106, 7729.

28 H. R. Moon, J. H. Kim and M. P. Suh, Angew. Chem. Int. Ed., 2005, 44, 1261.

29 M. P. Suh, H. R. Moon, E. Y. Lee, and S. Y. Jang, J. Am. Chem. Soc. 2006, 128, 4710.

30 Y. E. Cheon and M. P. Suh, Chem. Eur. J., 2008, 14, 3961.

31 S. Hermes, M.-K. Schröter, R. Schmid, L. Khodeir, M. Muhler, A. Tissler, R. W. Fischer and R. A. Fischer, Angew. Chem. Int. Ed., 2005, 44, 6237.

11032 F. Schröder, D. Esken, M. Cokoja, M. W. E. Van den Berg, O. I. Lebedev, G. Van Tendeloo, B. Walaszek, G. Buntkowsky, H.-H. Limbach, B. Chaudret and R. A. Fischer, J. Am. Chem. Soc., 2008, 130, 6119 .

33 M. Müller, S. Hermes, K. Kähler, M. W. E. Van den Berg, M. Muhler and R. A. Fischer, Chem. Mater., 2008, 20, 4576.

34 M. Sabo, A. Hensche, H. Froede, E. Klemm and S. Kaskel, J. Mater. Chem., 2007, 17, 3827.

35 S. Hermes, F. Schröder, R. Chelmowski, C. Wöll and R. A. Fischer, J. Am. Chem. Soc., 2005, 127, 13744.

12036 S. Turner, O. I. Lebedev, F. Schröder, D. Esken, R. A. Fischer, G. Van Tendeloo, Chem. Mater., 2008, 20, 5622.

37 Y. K. Hwang, D.-Y. Hong, J.-S. Chang, S. H. Jhung, Y.-K. Seo, J. Kim, A. Vimont, M. Daturi, C. Serre and G. Férey, Angew. Chem. Int. Ed., 2008, 47, 4144.

12538 T. Uemura, D. Hiramatsu, K. Yoshida, S. Isoda and S. Kitagawa, J. Am. Chem. Soc., 2008, 130, 9216. 